\title{
A NEW REVERSE ISOPERIMETRIC INEQUALITY AND ITS STABILITY
}

\author{
XIANG GAO
}

Abstract. In this paper, we deal with the reverse isoperimetric inequality for a closed and strictly convex curve in the Euclidean plane $\mathbb{R}^{2}$ involving the following geometric functionals associated to the given convex curve: length, areas of the region respectively included by the curve and the locus of curvature centers, and the integral of the radius of curvature. In fact, a stronger and sharp version of the reverse isoperimetric inequality proved by Pan and Yang in [1] is established with a simple Fourier series proof. Furthermore, we investigate the stability property of such an inequality (almost equality implies that the curve is nearly circular).

Mathematics subject classification (2010): Primary 52A38; Secondary 52A40. Keywords and phrases: isoperimetric inequality, Fourier series, stability.

\section{REFERENCES}

[1] S. L. PAN, J. N. YANG, On a non-local perimeter-preserving curve evolution problem for convex plane curves, Manuscripta Math. 127 (2008), 469-484.

[2] J. STEINER, Sur le maximum et le minimum des figures dans le plan, sur la sphère, et dans l'espace en général, I and II, J. Reine Angew. Math. (Crelle) 24 (1842), 93-152 and 189-250.

[3] S. L. PAN, H. ZhANG, A reverse isoperimetric inequality for convex plane curves, Beitrage Algebra Geom. 48 (2007), 303-308.

[4] H. Groemer, Geometric applications of Fourier series and spherical harmonics, Encyclopedia of Mathematics and its Applications, 61. Cambridge University Press, Cambridge, 1996.

[5] S. L. PAn, H. P. XU, Stability of a reverse isoperimetric inequality, J. Math. Anal. Appl. 350 (2009), $348-353$. 